\title{
Understanding the giant gamma-ray outburst on June 16, 2015 from the blazar $3 \mathrm{C} 279$
}

\author{
K. K. Singh \\ Physics Department, University of the Free State, Bloemfontein, South Africa 9301 and \\ Astrophysical Sciences Division, Bhabha Atomic Research Centre, Mumbai, India 400085 \\ P. J. Meintjes, F. A. Ramamonjisoa \\ Physics Department, University of the Free State, Bloemfontein, South Africa 9301 \\ kksastro@barc.gov.in
}

Received __; accepted _ 


\begin{abstract}
A very bright and fast varying gamma-ray flare has been detected from the blazar 3C 279 on June 16, 2015. We have studied the broadband spectral energy distribution of the source during the flaring episode and in the low activity state using a simple one zone leptonic model. We find that an electron energy distribution described by a broken power law can be used to reproduce the broadband emissions during the high and low activity states. The flux measurements at radio, infrared and optical frequencies are reproduced by the synchrotron emission resulting from the relativistic electrons in a jet magnetic field strength of 0.37 G. The gamma-ray emission from the blazar 3C 279 is attributed to the Comptonization of the IR seed photons from the dusty torus with a temperature of $870 \mathrm{~K}$. The outburst from the source observed on June 16, 2015 can be ascribed to an efficient acceleration process associated with a sudden enhancement in the electron energy density in the emitting region with respect to the low activity state. The fast gamma-ray variability at a minute timescale implies that the emission during the flare originates from a more compact region and the size of the emission zone in the low activity state is found to be four times larger than that during the flare. We have also used the model parameters derived from the broadband spectral energy distribution modelling to investigate a few physical properties of the jet during the outburst.
\end{abstract}

Subject headings: Galaxies: active - Flat Spectrum Radio Quasars: individual (3C 279) — gamma rays: general — radiation mechanism: nonthermal 


\section{Introduction}

The unification scheme of radio-loud active galactic nuclei suggests that blazars exhibit a pair of relativistic jets oriented in the opposite directions perpendicular to the accretion disc and one of them is inclined at small viewing angles $\left(\leq 10^{\circ}\right)$ to our line of sight (Urry \& Padovani 1995; Padovani et al. 2017). They are powered by accretion of matter from the surrounding gas onto supermassive black holes (SMBH) at the centre of their elliptical host galaxies. The relativistic jets transport matter in the form of a highly collimated plasma expelled from the nucleus of the host galaxies and are extended up to Mpc scales into the intergalactic space. The launching and evolution of these powerful relativistic plasma jets are not completely understood, however, it is generally believed that they are powered either by the rotational energy of a spinning SMBH at the centre through the Blandford-Znajek process or by the gravitational energy of the accreting matter (Blandford \& Znajek 1977). The energy flow is dissipated through the multi-wavelength emission downstream along the jet. The broadband non-thermal emissions from the plasma jets are amplified due to the relativistic Doppler boosting. The low energy emission observed at radio-optical and X-ray energies is attributed to the synchrotron radiation due to the relativistic electrons in the jet magnetic field. The high energy emission from blazars is still under debate and different scenarios have been proposed to explain the observed $\gamma$-ray spectral features (Böttcher et al. 2013).

In the leptonic processes, $\gamma$-ray photons are produced by the inverse Compton (IC) scattering of low energy seed photons by the relativistic electrons in the blazar jet (Dermer) 1995; Dermer et al. 2009). The energy of the target photon determines the dominance of Comptonization in the Thomson or Klein-Nishina (K-N) regime. If $\gamma$-rays are produced by the IC scattering in the Thomson regime, their spectral shape is straightforward related to the energy distribution of relativistic electrons in the emitting region. Whereas, the relation 
between the observed $\gamma$-ray spectrum and energy distribution of electrons is very complex if the $\gamma$-ray production takes place in the $\mathrm{K}-\mathrm{N}$ regime. Therefore, the spectral shapes of the observed high energy $\gamma$-rays from blazars are an indirect probe of the particle acceleration mechanisms in the jet, which have not been properly understood. The leptonic models with different forms of electron energy distribution cooling down in various populations of low energy target photons (synchrotron photons produced in the emission region, accretion disc photons reprocessed by the broadline region and dusty torus external to the jet) are widely used to model the broadband spectral energy distribution (SED) of most blazars (Böttcher et al. 2013; Abdo et al. 2010a; Agudo 2011; Singh et al. 2018). Hadronic processes including proton synchrotron, muon synchrotron, pion decay and cascade emission are also invoked to model the $\gamma$-ray emission from some blazars (Aharonian 2002; Böttcher 2009; Cerruti 2015). Recent detection of astrophysical neutrino events from the direction of the blazar TXS $0506+056$ provides additional evidence for the hadronic processes involved in the $\gamma$-ray production (Aartsen et al. 2018a, b ). The $p-p$ interactions are generally not considered in the hadronic processes for $\gamma$-ray emission from blazars due to a low particle density in the emitting zone. In a quite different scenario, the high energy $\gamma$-rays are not produced in the blazar jet but in the intergalactic space by the protons (components of ultra high energy cosmic rays accelerated in the jet) escaping from the emission region and beamed in the direction of the observer. These energetic protons initiate an electromagnetic cascade in the intergalactic space via interaction with low energy photons from the extragalactic background light (EBL) or cosmic microwave background radiation (CMBR) through photo-meson and pair-creation processes (Essey et al. 2011; Murase et al. 2012; Takami et al. 2013). The high energy $\gamma$-ray photons are produced by the electromagnetic cascade and propagate towards the Earth without any significant attenuation. This is known as hadron beam scenario and has been used to explain the $\gamma$-ray emission from a sample of hard spectra blazars having extreme properties (Tavecchio et al. 
2019; Singh et al. 2019a).

Multi-wavelength emissions from the blazars are observed to exhibit a rapid variability on minute timescales during the flaring episodes associated with a dramatic increase in the luminosity (Albert et al. 2007; Singh et al. 2012, 2015; Bartoli et al. 2016; Singh et al. 2019b) and a significant drop in the degree of optical polarization (Abdo et al. 2010b; Singh et al. 2019c). The behaviour of the spectral and temporal changes during near simultaneous multi-wavelength or orphan flares is found to be erratic and has yet not been understood. Various time-dependent single-zone emission models are used to explain the observed properties of outbursts from the blazars (Chen et al. 2011; Mastichiadis et al. 2013; Singh et al. 2017). Multi-zone emission models have also been proposed to explain the stochastic nature of the variability during the flaring activity of blazars (Zhang et al. 2016). Both single-zone and multi-zone models involving leptonic and hadronic processes are extensively used to study the flares depending on the observed properties of a source. However, a clear understanding of the physical process is lacking. Therefore, exploring the physical mechanism involved in the non-thermal emission during the flaring activity of blazars remains an important and major task in the blazar research today. In this work, we focus on the historical $\gamma$-ray flare observed from the blazar 3C 279 on June 16, 2015. 3C 279 is one of the most distant blazars at a cosmological redshift $z=0.536$ and belongs to the flat spectrum radio quasar (FSRQ) subclass of the blazars (Burbidge \& Rosenberg 1965). The FSRQ type blazars are characterized by the strong broad emission lines in the optical spectrum and a bright accretion disc due to high accretion rate. The high energy radiation from FSRQs is generally produced by IC scattering of the external seed photons emitted from the bright accretion disc and reprocessed by the broadline region and extended dusty torus surrounding the central SMBH. In the present work, we use IR photons from the dusty torus to reproduce the observed $\gamma$-ray emission from the FSRQ blazar 3C 279 during the giant outburst on June 16, 2015. In Section 2, we briefly summarize the important 
results from the June 2015 flare of 3C 279. The multi-wavelength data set and daily light curve are described in Section 3 and Section 4 respectively. In Section 5, we explain the single zone leptonic model to reproduce the broadband SEDs of the source. Finally, we discuss the important findings in Section 6 and conclude the study in Section 7. We have adopted the cosmological parameters $\mathrm{H}_{0}=70 \mathrm{~km} \mathrm{~s}^{-1} \mathrm{Mpc}^{-1}, \Omega_{m}=0.27$ and $\Omega_{\Lambda}=0.73$ from the $\Lambda$ CDM model.

\section{Summary of June 2015 outburst from 3C 279}

A giant outburst from the blazar 3C 279 was detected by the Fermi-Large Area Telescope (LAT) with the peak flux level of $(3.6 \pm 0.2) \times 10^{-5} \mathrm{ph} \mathrm{cm}^{-2} \mathrm{~s}^{-1}$ above $100 \mathrm{MeV}$

on June 16, 2015 (Ackermann et al. 2016). It was found historically to be the highest $\gamma$-ray flux measured from 3C 279 with an isotropic luminosity close to $10^{49} \mathrm{erg} \mathrm{s}^{-1}$. This active phase of the source allowed the investigation of $\gamma$-ray variability on minute-scale with a flux doubling time of $\sim 5$ minutes. This suggests a very compact emission region in the jet of $3 \mathrm{C}$ 279. The high energy $\gamma$-ray spectrum measured by LAT during the flaring activity of the source was more favorably described by a log-parabola model with spectral and curvature indices of $1.71 \pm 0.09$ and $0.20 \pm 0.05$ respectively than the power law model having a spectral index of 2.01 \pm 0.05 . The highest energy of photons detected was $56 \mathrm{GeV}$ with a probability of $99.99 \%$ at the end of the flare from 3C 279. During the flare, the high energy component of the SED peaks at energies between $0.3 \mathrm{GeV}$ and $1 \mathrm{GeV}$, whereas the peak energy is $1 \mathrm{GeV}$ at the beginning and end of the flare due to relatively hard spectra. This is not consistent with the previous emission states of 3C 279 when the peak energy in SED has been observed below $0.1 \mathrm{GeV}$. The instruments on board the AGILE satellite also detected intense $\gamma$-ray emission in the energy range $30 \mathrm{MeV}-50 \mathrm{GeV}$ from $3 \mathrm{C} 279$ during the outburst (Pittori et al. 2018). The high energy $\gamma$-ray flux measured by AGILE above $100 \mathrm{MeV}$ was 
$(1.3 \pm 0.13) \times 10^{-5} \mathrm{ph} \mathrm{cm}^{-2} \mathrm{~s}^{-1}$ with a power law photon spectral index of $2.14 \pm 0.11$ during the flare. The results obtained from AGILE observations during the high activity state of the blazar 3C 279 are compatible with the Fermi-LAT values within statistical uncertainty. The high energy $\gamma$-ray flare was also followed up with the ground based H.E.S.S. telescopes and resulted in a signal with $8.7 \sigma$ statistical significance above an energy threshold of 66 $\mathrm{GeV}$ in $\sim 2.2$ hours of observation (H. E. S. S. Collaboration 2019). The photon spectrum observed by H.E.S.S. telescopes was described by a soft power law with a spectral index of $4.2 \pm 0.3$ and the average integral flux above $200 \mathrm{GeV}$ was found to be $(7.6 \pm 0.7) \times 10^{-12} \mathrm{ph}$ $\mathrm{cm}^{-2} \mathrm{~s}^{-1}$. No indication of statistically significant variability was detected in the light curve and the flux points above $100 \mathrm{GeV}$ were consistent with an average flux level of $(6.5 \pm 0.6) \times$

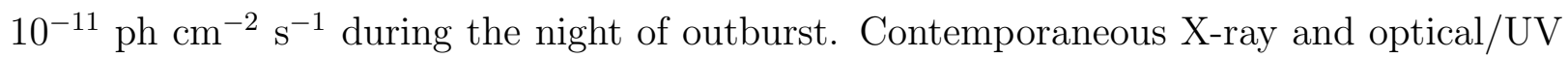
observations also hint for the flaring activity of 3C 279 at lower energies (Pittori et al. 2018; H. E. S. S. Collaboration 2019). The X-ray flux measured by the X-ray Telescope (XRT) on board the Neil Gehrels Swift observatory in the energy range 0.3-10 keV during the peak was about 4 times the flux level in low activity state of the source with a correlated variability in high energy $\gamma$-rays. The optical emission in $\mathrm{R}$ band was also observed to increase by $40 \%$ during the outburst (․․ E. S. S. Collaboration 2019).

\section{Data Set}

As discussed in Section 2, the high energy $\gamma$-ray emission from the blazar 3C 279 started to increase from June 14, 2015, attained a peak on June 16, 2015 and finally decreased to a low activity state on June 17, 2015. Therefore, we have used near simultaneous broadband data available from various space and ground based observations of the blazar 3C 279 during June 1-30, 2015 for the SED modelling. We have divided the whole period into three epochs: Pre-flare (June 1-13, 2015), Flare (June 16, 2015) and Post-flare (June 18-30, 
2015). We have also obtained the multi-wavelength light curves from high energy $\gamma$-ray to radio between May 1, 2015 and July 31, 2015 (MJD 57143-57234) to study the emission behaviour of the source in different energy bands for a longer period. The details of the multi-wavelength dataset used in this study are described below.

\section{1. $\gamma$-ray}

We have analyzed high energy $\gamma$-ray data from the Fermi-LAT observations in the energy range $100 \mathrm{MeV}$ - $500 \mathrm{GeV}$ using standard Fermitools (Fermi 1.0.1). The LAT is a pair-conversion telescope which directly detects $\gamma$-rays in the energy range from $20 \mathrm{MeV}$ to more than $1 \mathrm{TeV}$ (Atwood et al. 2009; Ajello et al. 2017). The publicly available Pass8 event data on blazar 3C 279 was downloaded from the Fermi-LAT data server 1 . We have only selected source class events with evclass $=128$ and evtype $=3$ in a region of interest (ROI) of radius $10^{\circ}$ centered at the position of 3C 279 (4FGL J1256.1-0547). All point sources listed in the 4FGL catalog (gll_psc_v18.fits) and located within $20^{\circ}$ radius from the center of ROI along with the Galactic (gll_iem_v06.fits) and extragalactic isotropic diffuse emission (iso_P8R3_SOURCE_V2.txt) for the background subtraction are included in the model file for an unbinned likelihood analysis. We have produced the time averaged spectral flux points in the energy range $100 \mathrm{MeV}-500 \mathrm{GeV}$ for the three epochs of 3C 279 observations using gtlike. The source 3C 279 is modelled with a log-parabola spectrum whereas the spectra of all the point sources in the ROI have been kept the same as in the 4FGL catalog (The Fermi-LAT Collaboration 2019).

\footnotetext{
${ }^{1}$ https://fermi.gsfc.nasa.gov/cgi-bin/ssc/LAT/LATDataQuery.cgi
} 


\subsection{X-ray}

The archival X-ray data available during three epochs in the energy bands $15-50 \mathrm{keV}$ and 2-20 keV have been obtained from the Burst Alert Telescope (BAT) and Monitor of All-sky X-ray Image (MAXI) observations of the blazar 3C 279 respectively. The BAT transient monitor (Krimm et al. 2013) on board the Neil Gehrels Swift observatory provides online data from the daily observations of the transient events in the sky2. We have estimated the time averaged flux points in the energy range $15-50 \mathrm{keV}$ for three epochs of 3C 279 observations. The instruments on the MAXI experiment also detect the X-ray transient events and monitor the intensity fluctuations of the known sources in the energy range 2-20 keV (Matsuoka et al. 2009). We have used MAXI on-demand proces\$3 to get the data for the blazar 3C 279 in two energy bands $2 \mathrm{keV}-6 \mathrm{keV}$ and $6 \mathrm{keV}-20 \mathrm{keV}$. Thus data from MAXI and BAT observations provide three spectral points for the X-ray component of the broadband SED of the source.

\subsection{Optical and Radio}

The optical/IR contemporaneous data during three epochs have been obtained from the Small \& Moderate Aperture Research Telescope System (SMARTS) project 4. The SMARTS project provides daily observations of selected blazars using two small aperture telescopes in three optical $(\mathrm{B}, \mathrm{V} \& \mathrm{R})$ and two infrared $(\mathrm{J} \& \mathrm{~K})$ bands. The details of data reduction and analysis are described in (Bonning et al. 2012). We have used the publicly available data in magnitude scale to get the energy flux points in different bands following

\footnotetext{
${ }^{2}$ https://swift.gsfc.nasa.gov/results/transients

${ }^{3}$ http://maxi.riken.jp/mxondem

${ }^{4}$ http://www.astro.yale.edu/smarts
} 
the conversion described in (Hayashida et al. 2015). The radio data at $15 \mathrm{GHz}$ are obtained from the $40 \mathrm{~m}$ telescope at Owens Valley Radio Observatory (OVRO) 5 . The details of the radio monitoring program using the $40 \mathrm{~m}$ telescope are described in (Richards et al. 2011). We have used the publicly available data of the blazar 3C 279 during the above period.

\section{Multi-wavelength Light Curve}

The multi-wavelength light curve of the blazar 3C 279 during May 1, 2015 - July 31, 2015 (MJD 57143-57234) is shown in Figure 1(a-f). The high energy $\gamma$-ray flux points in Figure 1(a) correspond to the one day binning of the Fermi-LAT data in the energy range $0.1-500 \mathrm{GeV}$. All the flux values have been derived at a statistical significance of $3 \sigma$ and above assuming a power law spectrum of the source. The X-ray data points plotted in Figure 1(b \& c) are obtained from the daily Swift-BAT and MAXI observations in the energy range $15-50 \mathrm{keV}$ and $2-20 \mathrm{keV}$ respectively. The error bars in both the light curves correspond to the statistical uncertainty at $1 \sigma$ level. Near simultaneous daily flux measurements in the optical/IR (B, V, R, J \& K) bands and at $15 \mathrm{GHz}$ radio from the SMARTS and OVRO observations are reported in Figure 1(d-f) respectively. A visual inspection of the one day binned multi-wavelength light curve suggests that the flaring activity of the blazar 3C 279 during June 14-16, 2015 (MJD 57187-57189) is more prominent in the high energy $\gamma$-ray band than in the remaining lower energy bands. In fact, the X-ray emissions in the $15-50 \mathrm{keV}$ and 2-20 keV energy bands do not show any noticeable change during the $\gamma$-ray outburst. The optical/IR light curves indicate an increasing activity in the emission level in all five bands $(\mathrm{B}, \mathrm{V}, \mathrm{R}, \mathrm{J} \& \mathrm{~K})$ during the $\gamma$-ray flaring episode. The radio emission measured at $15 \mathrm{GHz}$ is consistent with the low activity state of the

\footnotetext{
${ }^{5}$ http://www.astro.caltech.edu/ovroblazars
} 
source. Therefore, we observe that the giant $\gamma$-ray flaring activity of the FSRQ 3C 279 during June 14-16, 2015 (MJD 57187-57189) with a peak on June 16, 2015 (MJD 57189) is accompanied by a simultaneous increase in the optical/IR emission level. In order to quantify the degree of multi-band cross correlation, we have estimated the Spearman's rank correlation coefficient $\left(r_{s}\right)$ using simultaneous flux measurements in two different energy bands. For high energy $\gamma$-ray and optical/IR (B, V, R, J \& K) light curves, the estimated values of $r_{s}$ are in the range 0.60-0.72 at a statistical significance level of more than $95 \%$. This implies a strong positive correlation between $\gamma$-ray and optical/IR emissions from the blazar 3C 279. The high energy $\gamma$-ray flux points show a weak correlation $\left(r_{s}=0.15-0.21\right)$ with X-ray emission in 15-50 keV and 2-20 keV bands only 50\% statistical significance level. The correlation between X-ray and optical/IR emissions is also found to be weak. The radio emission is observed to be very weakly correlated $\left(r_{s}=0.08\right)$ with the high energy $\gamma$-ray flux measurements. Therefore, the correlation analysis based on the Spearman's rank correlation suggest that the high energy $\gamma$-ray and optical/IR emissions from the FSRQ 3C 279 are physically connected. This also hints support for the leptonic emission model for the blazars.

\section{Spectral Energy Distribution Modelling}

We have invoked a simple one zone leptonic model to reproduce the broadband emissions observed from the blazar 3C 279 during the period June 1-30, 2015. In this model (Massaro et al. 2006; Tramacere et al. 2009, 2011), the non-thermal emission is assumed to originate from a spherical blob of radius $R$ moving down the jet with a bulk Lorentz factor $\Gamma$ and entangled with a uniform magnetic field of strength $B$. The relativistic motion of the emitting blob leads to the strong Doppler boosting of the observed radiation due to 
the beaming effect. The relativistic Doppler factor $\delta$ is given by

$$
\delta=\frac{1}{\Gamma(1-\beta \cos \theta)}
$$

where $\theta$ is the viewing angle with respect to the line of sight of the observer and $\beta$ is the bulk speed of the blob in units of the speed of light $c$ in vacuum. Under the small viewing angle approximation for blazars $\left(\theta \sim 5^{\circ}\right.$ for $\left.3 \mathrm{C} 279\right), \delta \approx \Gamma$. The blob is homogeneously filled with the plasma of relativistic leptons $\left(e^{ \pm}\right)$and cold protons (expected to be present for providing charge neutrality). The protons are assumed to be at rest in the comoving frame and only relativistic leptons contribute to the non-thermal emission of the blob. The electrons are accelerated to relativistic energies through putative acceleration processes (e.g Fermi first and second order) and then injected to the blob. The electrons in the blob cool via the radiative processes namely synchrotron emission and inverse Compton scattering. The differential electron distribution is given by a broken power law, which can be expressed as

$$
\begin{aligned}
n(\gamma) & =N K \gamma^{-p}, \gamma_{\min } \leq \gamma \leq \gamma_{b} \\
& =N K \gamma_{b}^{q-p} \gamma^{-q}, \gamma_{b} \leq \gamma \leq \gamma_{\max }
\end{aligned}
$$

where $N$ and $\gamma\left(=\frac{E_{e}}{m_{e} c^{2}}\right)$ are the number density and Lorentz factor of the electrons respectively. $p$ and $q$ are the low and high energy spectral indices respectively. $\gamma_{\min }, \gamma_{b}$ and $\gamma_{\max }$ are the Lorentz factors corresponding to the minimum, break and maximum energies of the electron population. The electron distribution is normalized through the constant $K$ as

$$
\int_{\gamma_{\min }}^{\gamma_{\max }} \frac{n(\gamma)}{N} d \gamma=1
$$

The broadband emission from the blazar 3C 279 observed at radio and optical frequencies is dominated by the synchrotron radiation of electrons described by Equation (2) interacting with the tangled magnetic field of the blob. Whereas, the high energy emission at X-ray and $\gamma$-ray energies is reproduced by Comptonization of the seed photons originating from the dusty torus (dust present in the cold molecular gas). The dusty torus (DT) is considered as 
an isotropic structure obscuring the accretion disc surrounding the SMBH. This is expected to survive at locations with ambient temperature below $1500 \mathrm{~K}$ surrounding the blazar. The location of DT from the central SMBH $\left(R_{D T}\right)$ can be expressed as (Sikora et al. 2002)

$$
R_{D T} \approx \frac{1}{T_{D}^{2}}\left(\frac{L_{d i s c}}{4 \pi \sigma_{S B}}\right)^{1 / 2}
$$

where $T_{D}, L_{\text {disc }}$ and $\sigma_{S B}$ are the dust temperature, accretion disc luminosity and Stefan-Boltzmann constant respectively. The dust is heated by the thermal emission at UV/optical wavelengths from the accretion disc and re-emits at IR wavelengths. The disc emission reprocessed by DT (IR photons) acts like an external radiation field for the inverse Compton scattering of the seed photons by the relativistic electrons described by Equation (2). The Comptonization of IR seed photons emitted from the DT is assumed to take place in the Thomson regime. Assuming that the intensity of the radiation produced by synchrotron $\left(I_{s y n}\right)$ and inverse Compton $\left(I_{I C}\right)$ processes is isotropic, the total observed flux is calculated with (Begelman et al. 1984)

$$
F_{o b s}\left(\nu_{o b s}\right)=\frac{\delta^{3}(1+z) V}{d_{L}^{2}}\left(I_{s y n}(\nu)+I_{I C}(\nu)\right)
$$

where $V$ is the volume of the emission region and $d_{L}$ is the luminosity distance (in $\Lambda$ CDM model) of the source at redshift $z$. The observed frequency $\nu_{\text {obs }}$ of the radiation is related to the emitted frequency $\nu$ as

$$
\nu_{o b s}=\frac{\delta \nu}{1+z}
$$

This simple one zone leptonic model for the blazar emission uses $R, z, \Gamma, \theta, B, N, \gamma_{\min }$, $\gamma_{\max }, p, q, L_{d i s c}, R_{D T}$ and $T_{D}$ as input parameters. These parameters sufficiently specify the properties of non-thermal broadband emission region and electron energy distribution in the jet. Therefore, the observed multi-wavelength data can be compared with the estimated non-thermal flux values from the model. We have employed the publicly available 
AGN-SED-tool (SSC/EC Simulator)6 to derive the best fit parameters for the broadband spectral energy distributions of the blazar 3C 279 during three epoch as described below.

\subsection{Pre-flare (June 1-13, 2015)}

The pre-flare epoch is defined to characterize the low activity state of the source prior to the flaring episode. For this epoch, we have used near simultaneous multi-wavelength observations available during June 1-13, 2015 from the OVRO, SMARTS, MAXI \& BAT and LAT. The time-averaged flux points obtained from these observations are shown in Figure 2. We have employed the one zone leptonic model described above to reproduce the radio, optical, X-ray and high energy $\gamma$-ray emissions during the pre-flare epoch. The model curves corresponding to the synchrotron and inverse Compton scattering of the external photons from the dusty torus (EC on DT) during the low activity state of 3C 279 are depicted in Figure 2, We observe that the model satisfactorily reproduces the time-averaged broadband flux measurements in the low activity state of the source. The best fit model parameters obtained from the SED modelling are given in column 3 of the Table 1 .

\section{2. $\quad$ Flare (June 16, 2015)}

The flare epoch corresponds to the period of a giant outburst of the blazar 3C 279 detected by the Fermi-LAT on June 16, 2015. We have obtained the contemporaneous data available from the SMARTS, MAXI \& BAT and LAT in the optical, X-ray and high energy $\gamma$-ray bands respectively. Fortunately, very high energy data from the H.E.S.S. observations are also available during the night of the flaring episode. The observed very high energy

\footnotetext{
${ }^{6}$ http://www.isdc.unige.ch/sedtool/PROD/SED.html
} 
above $66 \mathrm{GeV}$ is described by a power law of the form (H. E. S. S. Collaboration 2019)

$$
\frac{d N}{d E}=(2.5 \pm 0.5) \times 10^{-9}\left(\frac{E}{98 \mathrm{GeV}}\right)^{-4.2 \pm 0.3} \mathrm{ph} \mathrm{cm}^{-2} \mathrm{~s}^{-1} \mathrm{TeV}^{-1}
$$

We have derived the very high energy flux points at three energies $66 \mathrm{GeV}, 200 \mathrm{GeV}$, and $500 \mathrm{GeV}$ from the above relation using the error propagation method to construct the broadband SED of the source during the outburst. These flux points have also been corrected for absorption due to EBL via photon-photon pair production following the methodology described in (Singh et al. 2014) corresponding to the EBL model proposed by Franceschini et al. (2008). The flux points are converted to the $\nu F_{\nu}\left(\mathrm{erg} \mathrm{cm}^{-2} \mathrm{~s}^{-1}\right)$ values for the SED representation using the relation

$$
E^{2} \frac{d N}{d E} \equiv \nu F_{\nu}
$$

The contemporaneous multi-wavelength flux points along with the SED model curves are shown in Figure 3, It is evident from the figure that the leptonic one zone model reproduces the broadband emission from the FSRQ 3C 279 during the flaring activity very well. The corresponding best fit model parameters are reported in column 4 of the Table 1 .

\subsection{Post-flare (June 18-30, 2015)}

The flaring activity of the source decays to low emission state on June 17, 2015. Therefore, we have defined the post-flare epoch of the blazar 3C 279 during the period June 18-30, 2015 to characterize the low activity state of the source after the vanishing of the outburst. The broadband emissions measured in optical, X-ray and high energy $\gamma$-ray bands from the near simultaneous observations using SMARTS, MAXI \& BAT and LAT during the post-flare epoch are depicted in Figure 4. The model curves in Figure 4 for reproducing the broadband emission from 3C 279 are in good agreement with the measured 
flux points. The best fit parameters of the model corresponding to the post-flare epoch are summarized in column 5 of the Table 1.

\section{Discussion}

Near simultaneous observations of the giant $\gamma$-ray flaring activity of the blazar 3C 279 by the Fermi-LAT and H.E.S.S. telescopes on June 16, 2015 provide a very important data set for exploring the parameter space of the blazar emission model. The minimum variability timescale of $\sim 5$ minutes measured by the Fermi-LAT during the outburst of the source characterizes the blazar 3C 279 as one of the blazars with the fastest variability in the GeV-TeV energy regime (Ackermann et al. 2016; H. E. S. S. Collaboration 2019). We have used a simple one zone leptonic model to reproduce the non-thermal broadband emission from the source during low and high activity states. The sets of model parameters derived from the SED modelling (Table 1) represent one of the possible combinations for the source activity. A simple comparison of the individual model parameters obtained for three different epochs suggests that the electron number density $(N)$ in the emission region changes significantly during the flaring episode. However, the electron energy distribution is described by a broken power law with spectral indices $p \sim 2.2$ and $q \sim 3.7$ at low and high energies respectively. If the broken power law for electron energy distribution is simply attributed to the radiative cooling effects, the spectral indices should satisfy the condition $q=p+1$. A deviation from this condition indicates a more complex situation where different acceleration or cooling processes dominate in different energy bands. In the present study, the values of the spectral indices broadly satisfy the condition of radiative cooling break. The energy density of the radiating particles or electrons $\left(U_{e}\right)$ and magnetic field $\left(U_{B}\right)$ in the comoving frame can be estimated as

$$
U_{e}=\int_{\gamma_{\min }}^{\gamma_{\max }} m_{e} c^{2} \gamma n(\gamma) d \gamma
$$


and

$$
U_{B}=\frac{B^{2}}{8 \pi}
$$

From Table 1, the strength of the magnetic field in the emission region is found to be $\sim$ $0.37 \mathrm{G}$ during all the three epochs, whereas the electron number density attains a maximum value of $\sim 10^{4}$ during the flaring activity of the blazar $3 \mathrm{C} 279$. The estimated values of $U_{e}$ and $U_{B}$ for three epochs of the source activity are listed in Table 2. We observe that the particle and magnetic energy densities are near the equipartition in the low activity states, whereas during the flaring episode, the two quantities are far from the equipartition suggesting that the jet is particle or matter dominated. The flaring activity can be attributed to the sudden increase in the electron density in the emission region. Also, the size of the emission region during the outburst is smaller than that in the low activity states of the source before and after the flare (Table 1). This suggests that the non-thermal emission from the inner region of the blob with relatively higher electron energy density dominates the broadband emission measured during the $\gamma$-ray flaring activity of 3C 379 on June 16, 2015. The strong flaring activity due to the sudden increase in the particle energy density has also been modelled in individual blazars like Mrk 421 using a time dependent leptonic model (Singh et al. 2017). The equipartition condition between the radiating particle and magnetic field energy densities offers a minimum power solution to the blazar jet emission. It becomes highly uncertain when magnetic field energy density is coupled with the total baryonic energy density of the jet.

The synchrotron peak frequency $\nu_{p} \sim 3.2 \times 10^{13} \mathrm{~Hz}$ obtained from the SED modelling during the three epochs supports the classification of 3C 279 as a low-synchrotron-peaked blazar. The observed synchrotron peak frequency can be expressed in terms of the model parameters as

$$
\nu_{p}=\left(\frac{\delta}{1+z}\right) \frac{\gamma_{b}^{2} e B}{2 \pi m_{e} c}
$$

where $e$ is the electronic charge. The $\gamma$-ray emission from the source can be attributed to 
the inverse Compton scattering of the external photons originating from the dusty torus with a temperature of $870 \mathrm{~K}$ located at a distance of $\sim 6 \times 10^{19} \mathrm{~cm}$ from the central engine. This dusty torus reprocesses a fraction $(\sim 20 \%)$ of the accretion disc luminosity in the IR regime. The Comptonization of IR seed photons emitted from the dusty torus takes place in the Thomson regime. The non-thermal emissions from blazars demand very large energies and extreme powers associated with the relativistic jets. The physics of powering the jets in astrophysical systems like blazars hosting a SMBH at the center is not clearly understood. Based on the various parameters derived from the SED modelling of the blazar 3C 279 , the jet kinetic power $\left(P_{j}\right)$ in the stationary frame of the host galaxy of the source can be obtained from the relation (Celotti \& Ghisellini 2008)

$$
P_{j}=\pi R^{2} \Gamma^{2} c\left(U_{e}+U_{B}+U_{p}\right)
$$

where $U_{p}$ is the comoving energy density of the cold protons in the jet plasma which can be calculated as

$$
U_{p}=m_{p} c^{2} \int n(\gamma) d \gamma
$$

$m_{p}$ being the rest mass of the proton. The estimated values of the jet power and other associated quantities from the best fit model parameters are given in Table 2, It is observed that the jet power and radiative power $\left(P_{r}\right)$ corresponding to the non-thermal emission are highest during the flaring epoch. The radiative power is found to be less than the jet power, indicating that only a small fraction of the jet kinetic power is dissipated in the form of observed radiation. This is consistent with the general characteristics of the blazar jet emission. The Eddington luminosity for a $\mathrm{SMBH}$ of mass $M$ is given by

$$
L_{E d d}=1.25 \times 10^{38}\left(\frac{M}{M_{\odot}}\right) \mathrm{erg} \mathrm{s}^{-1}
$$

where $M_{\odot}$ is the solar mass. For the blazar 3C 279, $M$ is estimated to be in the range of $(3-8) \times 10^{8} \mathrm{M}_{\odot}($ Nilsson et al. 2009). Therefore, the maximum Eddington luminosity 
for $3 \mathrm{C} 279$ is $\sim 10^{47} \mathrm{erg} \mathrm{s}^{-1}$, which is larger than the jet power reported in Table 2 . This indicates that the non-thermal emission from 3C 279 takes place at sub-Eddington limit. The model parameter space obtained in the present study is broadly consistent with the previous values in the literature (Sahavanathan et al. 2018; Hayashida et al. 2015; Sahavanathan \& Godambe 2012). Recently, Shah et al. (2019) have studied the flaring episode of 3C 279 observed in January 2018 and report that the increase in the value of $\Gamma$ causes flux enhancement during the outburst. Böttcher et al. (2013) have shown that the hadronic models with a very large power in relativistic protons, high magnetic field strength and extreme jet power cannot satisfactorily fit the broadband SED of 3C 279 due to the spectral break at GeV energies in the observed spectrum. A clumpy jet model with strings of compact plasmoids is also proposed to interpret the bright and fast $\gamma$-ray flares of the blazar 3C 279 (Vittorini et al. 2017). In this model, low energy emission in optical-UV bands is attributed to the synchrotron radiation due to relativistic electrons accelerated around the plasmoids. The synchrotron radiation is partially reflected back by a string which acts like a moving mirror for the approaching companions. Resulting which, shrinking gap transient overdensities of seed photons are build up around the plasmoid and Comptonization of these seed photons produces high energy $\gamma$-rays during the flaring episodes (Vittorini et al. 2017).

\section{Conclusion}

In this paper, we study the broadband spectral energy distribution of 3C 279 using the multi-wavelength observations during June 1-30, 2015. We have produced the SEDs of the source for three epochs corresponding to the low and high activity states under the framework of a simple one zone leptonic model. We find that the non-thermal broadband emission from the jet of blazar 3C 279 during the whole period can be reproduced by an 
electron energy distribution described by a broken power law. During the low activity states of the source before and after the giant outburst on June 16, 2015, the magnetic and electron energy densities approximately satisfy the equipartition condition. The giant flaring activity in the source is caused by a sudden increase in the electron energy density in the inner region of the emitting blob. The size of the emission region during the flare is smaller than that in the low activity state by a factor of $\sim 4$. The enhancement in the electron energy density can be attributed to the transient injection of relativistic electrons in the inner region of the jet. At low energies, the radiation is produced by the synchrotron process, and inverse Compton scattering of the seed photons from the dusty torus dominates at X-ray and $\gamma$-ray energies. The high energy emission from 3C 279 due to the Comptonization of the IR photons from dusty torus indirectly confirms the presence of hot dust in blazars.

We thank the anonymous reviewer for his/her suggestions and comments to improve the contents of this study. This research has made use of data from the OVRO 40-m monitoring program (Richards, J. L. et al. 2011, ApJS, 194, 29) which is supported in part by NASA grants NNX08AW31G, NNX11A043G, and NNX14AQ89G and NSF grants AST-0808050 and AST-1109911. This paper has made use of up-to-date SMARTS optical/near-infrared light curves that are available at www.astro.yale.edu/smarts/glast/home.php. This research has made use of MAXI data provided by RIKEN, JAXA and the MAXI team. We acknowledge the use of Swift/BAT transient monitor results provided by the Swift/BAT team in this research. 


\section{REFERENCES}

Urry, C. M. \& Padovani, P. 1995, PASP, 107, 803

Padovani, P. et al. 2017, A\&ARv, 25, 2

Blandford, R. D. \& Znajek, R. L. 1977, MNRAS, 179, 433

Böttcher, M. et al. 2013, ApJ, 768, 54

Dermer, C. D. 1995, ApJ, 446, 63

Dermer, C. D. et al. 2009, ApJ, 692, 32

Abdo, A. A. et al. 2010a, ApJ, 716, 30

Agudo, I. 2011, ApJL, 735, 10

Singh, K. K. et al. 2018, APh, 103, 122

Aharonian, F. A. 2002, MNRAS, 332, 215

Böttcher, M. 2009, ApJ, 703, 1168

Cerruti, M. 2015, MNRAS, 448, 910

Aartsen, M. G. et al. 2018a, Science, 361, 1378

Aartsen, M. G. et al. 2018b, Science, 361, 147

Essey, W. et al. 2011, ApJ, 731, 51

Murase, K. et al. 2012, ApJ, 749, 63

Takami, H. et al. 2013, ApJL, 771, 32

Tavecchio, F. 2019, MNRAS, 483, 1802 
Singh, K. K. et al. 2019a, NewA, 73, 101278

Albert, J. et al. 2007, ApJ, 669, 862

Singh, K. K. et al. 2012, NewA, 17, 679

Singh, K. K. et al. 2015, APh, 61, 32

Bartoli, B. et al. 2016, ApJS, 222, 6

Singh, K. K. et al. 2019b, AdSpR, 63, 766

Abdo, A. A. et al. 2010b, Nature, 463, 919

Singh, K. K. et al. 2019c, Ap\&SS, 364, 88

Chen, X. et al. 2011, MNRAS, 416, 2368

Mastichiadis, A. et al. 2013, MNRAS, 434, 2684

Singh, K. K. et al. 2017, NewA, 54, 24

Zhang, H. et al. 2016, ApJ, 829, 69

Burbidge, E. M. \& Rosenberg, F. D. 1965, ApJ, 142, 1673

Ackermann, M. et al. 2016, ApJL, 824, 20

Pittori, C. et al. 2018, ApJ, 856, 99

H. E. S. S. Collaboration 2019, A\&A, 627, 159

Atwood, W. B. et al. 2009, ApJ, 697, 1071

Ajello, M. et al. 2017, ApJSS, 232, 18

The Fermi-LAT Collaboration 2019, arXiv:1902.10045 
Krimm, H. A. et al. 2013, ApJSS, 209, 14

Matsuoka, M. et al. 2009, PASJ, 61, 999

Bonning, E. et al. 2012, ApJ, 756, 13

Hayashida, M. et al. 2015, ApJ, 807, 79

Richards, J. L. et al. 2011, ApJSS, 194, 29

Massaro, E. et al. 2006, A\&A, 448, 861

Tramacere, A. et al. 2009, A\&A, 501, 879

Tramacere, A. et al. 2011, ApJ, 739, 66

Sikora, M. et al. 2002, ApJ, 577, 78

Begelman, M. C. et al. 1984, RvMP, 56, 255

Singh, K. K. et al. 2014, NewA, 27, 34

Franceschini, A. et al. 2008, A\&A, 487, 837

Celotti, A. \& Ghisellini, G. 2008, MNRAS, 385, 283

Nilsson, K. et al. 2009, A\&A, 505, 601

Sahayanathan, S. et al. 2018, RAA, 18, 035

Sahayanathan, S. \& Godambe, S. 2012, MNRAS, 419, 1660

Shah, Z. et al. 2019, MNRAS, 484, 3168

Böttcher, M. et al. 2013, ApJ, 768, 54

Vittorini, V. et al. 2017, ApJL, 843, L23 
This manuscript was prepared with the AAS IATEX macros v5.2. 


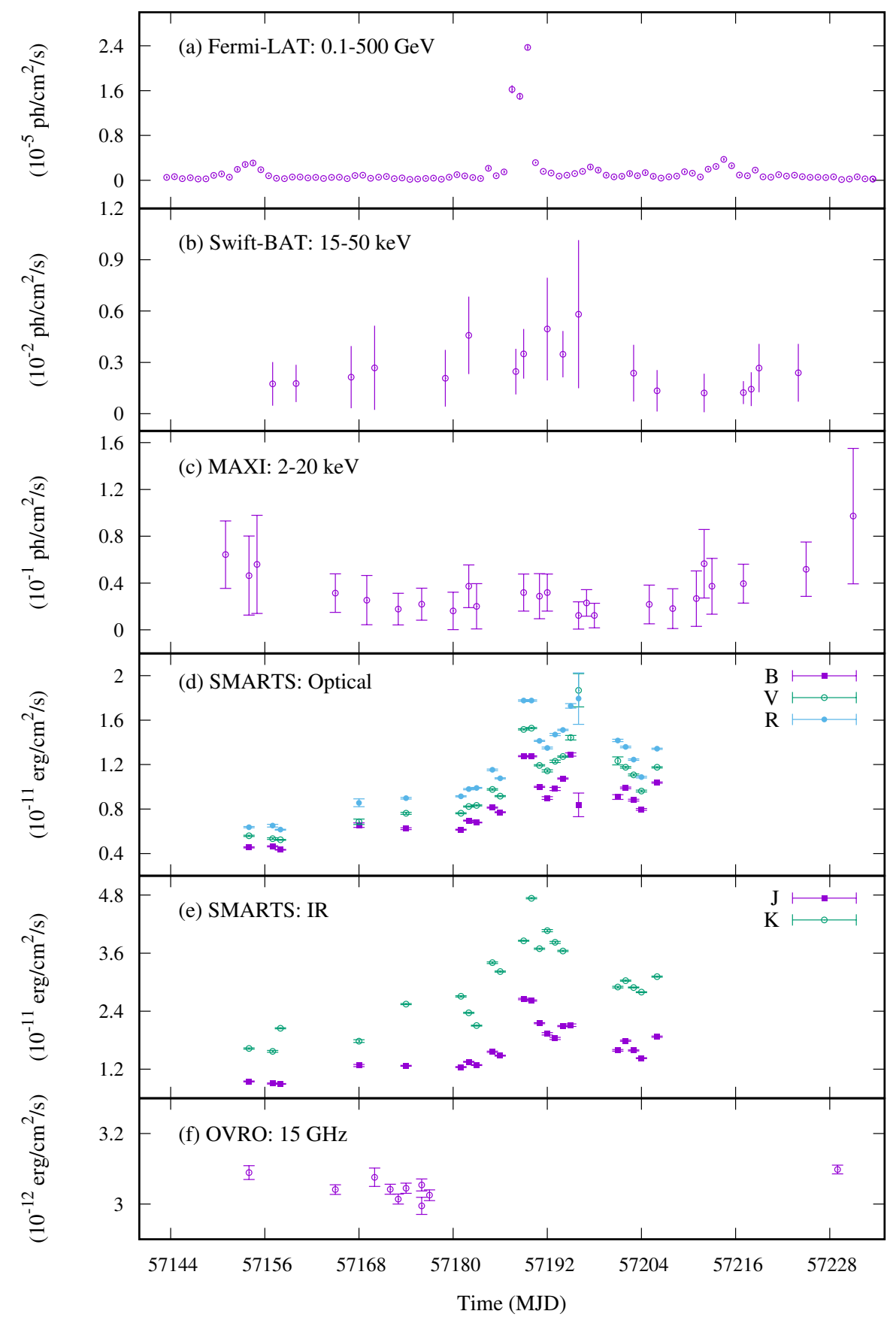

Fig. 1.- One day binned multi-wavelength light curve of the FSRQ 3C 279 during the period May 1, 2015 to July 31, 2015 (MJD 57143-57234) from the Fermi-LAT, Swift-BAT, MAXI, SMARTS and OVRO-40 m telescope observations. The giant $\gamma$-ray flaring episode peaks on June 16, 2015 (MJD 57189). 

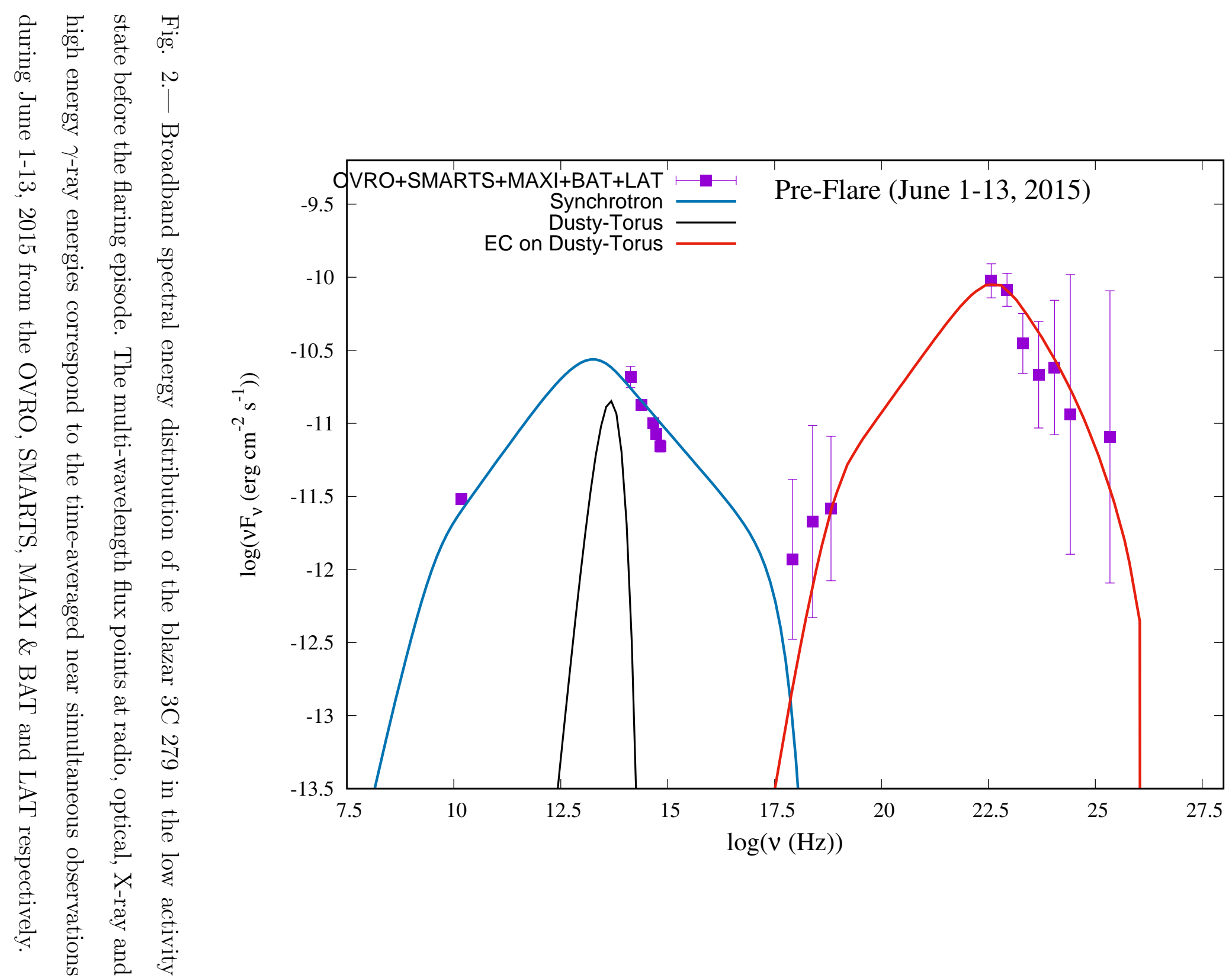


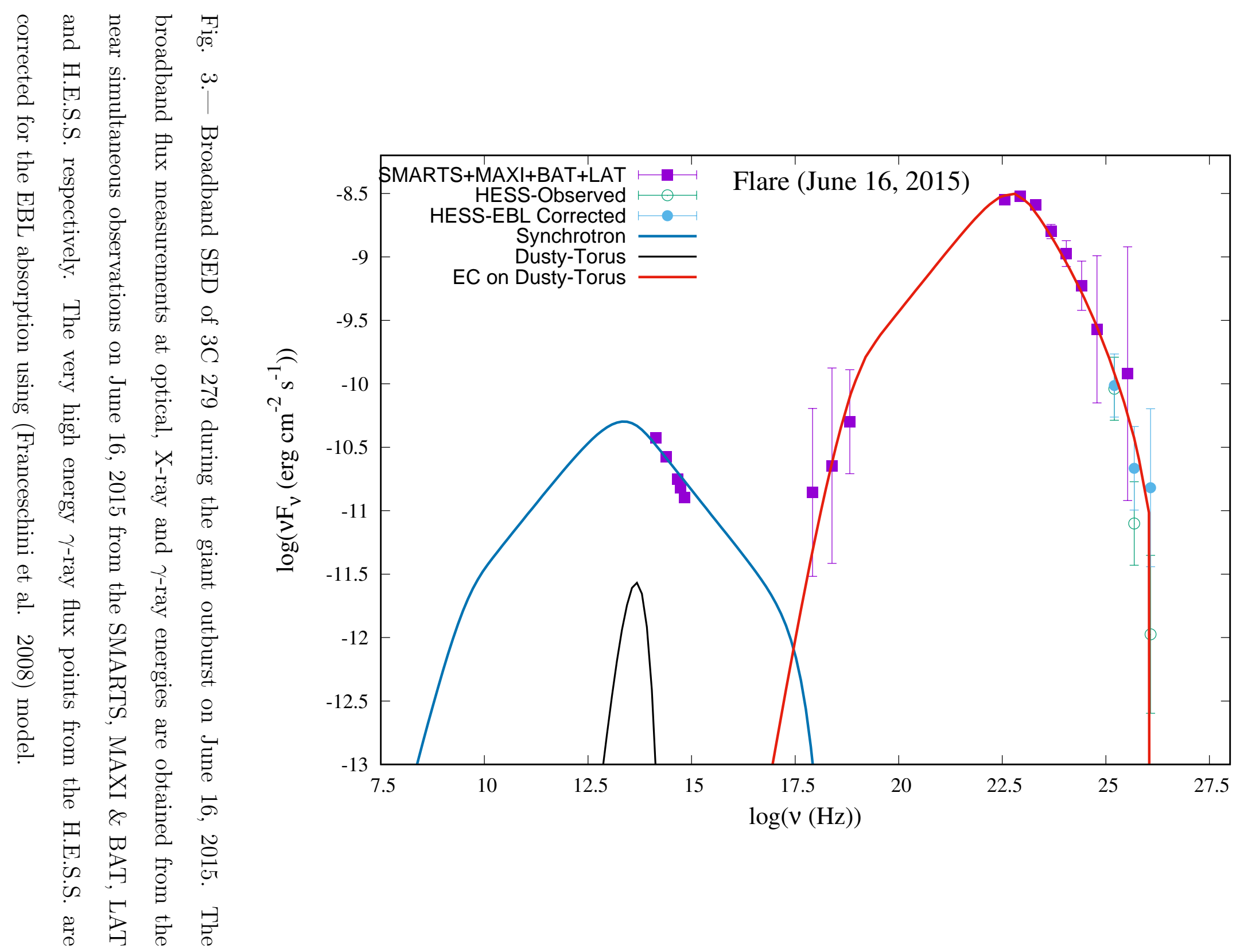




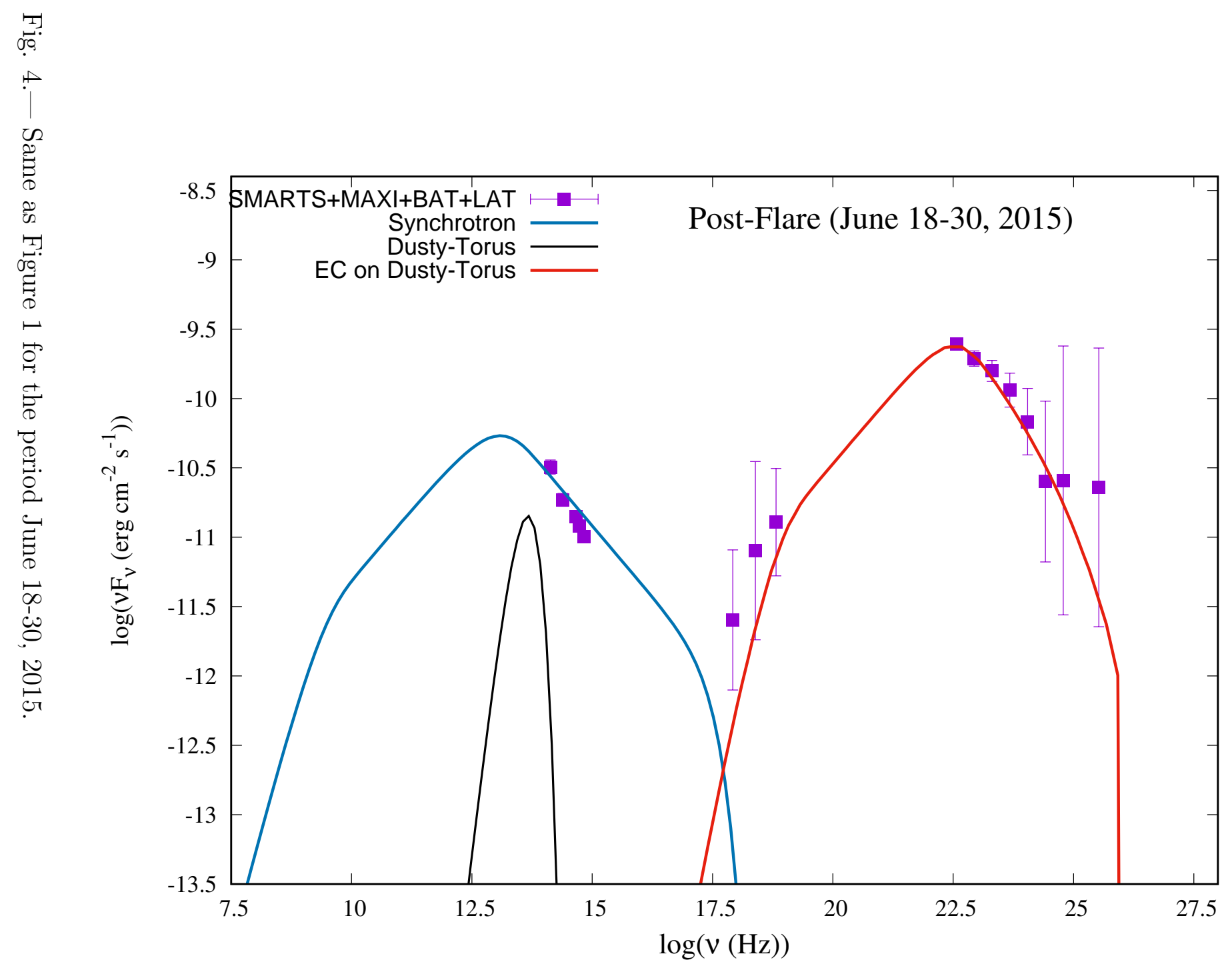


Table 1: Summary of the best fit parameters for the broadband spectral energy distributions of the FSRQ 3C 279 during three epochs from the simple one zone leptonic emission model for blazars.

\begin{tabular}{|c|c|c|c|c|}
\hline Parameters (Units) & Symbol & Pre-Flare & Flare & Post-Flare \\
\hline Redshift & $\mathrm{z}$ & 0.536 & 0.536 & 0.536 \\
\hline Size of emission region $(\mathrm{cm})$ & $\mathrm{R}$ & $9.3 \times 10^{16}$ & $2.1 \times 10^{16}$ & $9.3 \times 10^{16}$ \\
\hline Jet viewing angle $\left(^{\circ}\right)$ & $\theta$ & 2 & 2 & 2 \\
\hline Bulk Lorentz factor & $\Gamma$ & 21 & 21 & 21 \\
\hline Magnetic Field (G) & $\mathrm{B}$ & 0.37 & 0.37 & 0.37 \\
\hline Low energy spectral index & $\mathrm{p}$ & 2.18 & 2.18 & 2.18 \\
\hline High energy spectral index & $q$ & 3.68 & 3.82 & 3.82 \\
\hline Minimum electron Lorentz factor & $\gamma_{\min }$ & 10 & 10 & 10 \\
\hline Maximum electron Lorentz factor & $\gamma_{\max }$ & $10^{5}$ & $10^{5}$ & $10^{5}$ \\
\hline Electron Lorentz factor at break & $\gamma_{b}$ & 800 & 950 & 750 \\
\hline Electron number density $\left(\mathrm{cm}^{-3}\right)$ & $\mathrm{N}$ & 70 & $10^{4}$ & 200 \\
\hline Accretion disc luminosity $\left(\mathrm{erg} \mathrm{s}^{-1}\right)$ & $\mathrm{L}_{\text {disc }}$ & $1.9 \times 10^{46}$ & $1.9 \times 10^{46}$ & $1.9 \times 10^{46}$ \\
\hline Accretion disc temperature $(\mathrm{K})$ & $\mathrm{T}_{\text {disc }}$ & $10^{5}$ & $10^{5}$ & $10^{5}$ \\
\hline Location of dusty torus (cm) & $\mathrm{R}_{D T}$ & $6.1 \times 10^{19}$ & $6.1 \times 10^{19}$ & $6.1 \times 10^{19}$ \\
\hline Dust temperature (K) & $\mathrm{T}_{D}$ & 870 & 870 & 870 \\
\hline
\end{tabular}


Table 2: Few important source parameters derived from the spectral energy distribution modelling of the FSRQ 3C 279 during three epochs.

\begin{tabular}{|c|c|c|c|c|}
\hline Parameters (Units) & Symbol & Pre-Flare & Flare & Post-Flare \\
\hline Doppler factor & $\delta$ & 27 & 27 & 27 \\
\hline Electron energy density $\left(\mathrm{erg}_{\mathrm{cm}}^{-3}\right)$ & $\mathrm{U}_{e}$ & $2.2 \times 10^{-3}$ & $3.2 \times 10^{-1}$ & $6.3 \times 10^{-3}$ \\
\hline Proton energy density $\left(\mathrm{erg} \mathrm{cm}^{-3}\right)$ & $\mathrm{U}_{p}$ & $1.1 \times 10^{-2}$ & 1.5 & $3.0 \times 10^{-2}$ \\
\hline Magnetic energy density $\left(\mathrm{erg} \mathrm{cm}^{-3}\right)$ & $\mathrm{U}_{B}$ & $5.4 \times 10^{-3}$ & $5.4 \times 10^{-3}$ & $5.4 \times 10^{-3}$ \\
\hline Synchrotron peak frequency $(\mathrm{Hz})$ & $\nu_{p}$ & $3.2 \times 10^{13}$ & $3.2 \times 10^{13}$ & $3.2 \times 10^{13}$ \\
\hline Jet power $\left(\operatorname{erg~s}^{-1}\right)$ & $\mathrm{P}_{j}$ & $6.5 \times 10^{45}$ & $3.5 \times 10^{46}$ & $1.4 \times 10^{46}$ \\
\hline Radiative power $\left(\mathrm{erg} \mathrm{s}^{-1}\right)$ & $\mathrm{P}_{r}$ & $1.7 \times 10^{44}$ & $4.4 \times 10^{45}$ & $4.2 \times 10^{44}$ \\
\hline Total power $\left(\operatorname{erg~s}^{-1}\right)$ & $\mathrm{P}$ & $6.7 \times 10^{45}$ & $3.8 \times 10^{46}$ & $1.5 \times 10^{46}$ \\
\hline
\end{tabular}

